\title{
Outage Capacity and Energy Efficiency trade-off for Distributed MIMO Systems
}

\author{
Zi-Hao LI ${ }^{1, a^{*}}$,Gong-Bin QIAN ${ }^{1}$,Chun-Long HE ${ }^{1}$,Xing-Quan $\mathrm{LI}^{1}$ and Ce ZHANG ${ }^{1}$ \\ ${ }^{1}$ College of information Engineering,ShenZhen University,ShenZhen, China \\ aShendasidong@163.com
}

\begin{abstract}
Keywords:Outage capacity,Energy efficiency,Distributed MIMO systems.
Abstract.In this paper,we investigate the relationship between the outage capacity and the transmit power.Finally,we derived the energy efficiency(EE) based on outage capacity for the distributed multiple-input multiple-output(MIMO) systems under the idealistic power model and the realistic power model,respectively. Then we evaluate the EE performance under three parameters such as the cell radius,path loss exponent and outage probability.From theoretical analysis,we obtain that the outage capacity and the transmit power are not a simple linear relationship.Numerical results show that the performance of the EE is increased with the increase of the cell radius, the performance of the EE is decreased with the increase of the outage probability,and the performance of EE increase with the decrease of the path loss exponent.
\end{abstract}

\section{Introduction}

Due to improve system capacity, increase communication link stability and reduce the power consumption of the system, distributed antenna systems(DAS) has a great advantage and attracted more and more attention, and has been introduced in an earlier paper[1][2],Compared to the co-located multiple-inputmultiple-output(C-MIMO) technology[3][4].Distributed multiple-input multiple-output(D-MIMO) have micro diversity and macro diversity[5].

Outage probability and channel capacity are two important performance indexes in the upper level network planning of D-MIMO systems. The capacity can be divided into ergodic capacity and outage capacity.In the early work[6][7], the definition of ergodic mutual information were given. The expression of the approximated maximumrate and outage capacity have been derived in [8].

The research of energy efficiency(EE) is becoming more and more important[9].The ergodic capacity for energy efficiency research have been discussed in [10].The outage probability of D-MIMO systems have been introduced in [11].The reference [10][12] discussed the EE based on the ergodic capacity, and didn't discuss EE based on outage capacity.

On the basis of the above work[6][10][13], in this paper we first investigate the relationship between the outage capacity and transmit power,the performance of EE based on mutual information of outage capacity.Secondly discuss the three parameters such as the cell radius, the path loss and the outage probability for the EE which hope to find some factors that affect EE.

In this paper,there have some notations as follows. The $\mathrm{I}_{\mathrm{M}}$ denotes the identity matrix of size $\mathrm{k} \times$ $\mathrm{k}$. The operator $\mathrm{E}(\cdot)$ denotes expectation, $\mathrm{V}(\cdot)$ denotes variance, and the $\operatorname{erfc}(\cdot)$ denotes the error function.

\section{System Model}

We consider a D-MIMO systems with $N$ remote access units(RAUs).For the convenience,we only consider a single user scenario,the mobile stations(MSs) has $M$ antennas and each RAUs are equipped with $Q$ antennas.In this paper,the D-MIMO systems can be denoted by $(M, N, Q)$. When $N=1$, the D-MIMO systems becomesa C-MIMO systems. Specially, the central unit(CU) can be considered as a special RAU and is denoted by RAU 1 when the $Q=1$. Besides,assuming that the transmitter does not know the channel state information(CSI), but the reciever knows. The received signal at the BS of the downlink D-MIMO systems can be written as 


$$
\mathrm{Y}=\mathbf{H}(\mathrm{d}) \mathbf{X}+\mathbf{n},
$$

where $\mathrm{Y}$ is the $M \times 1$ received signal vector, $\mathrm{X}=\left[\mathrm{x}_{1}, \mathrm{x}_{2}, \ldots \mathrm{x}_{\mathrm{NL}}\right]$ isthe transmitted signal vector, $\mathrm{d}=\left[d_{1}, d_{2}, \ldots, d_{Q}\right]^{T}$ indicates the distance vector from the $N$-th RAU to MS, where $\mathbf{n}$ is the additive white zero-mean complex Gaussian noise with unit variance, $\mathbf{H}$ is the $M \times L N$ random matrix, which consist of the small-scale fast fading and large scale fading,can be expressed as [6]

$$
\mathrm{H}_{\mathrm{q}}\left(\mathbf{d}_{\mathbf{q}}\right)=h_{\mathrm{sh}, \mathrm{q}}, \mathrm{H}_{\mathrm{w}, \mathrm{q}}, 1 \leq \mathrm{q} \leq \mathrm{Q},
$$

where $\mathrm{H}_{\mathrm{w}, \mathrm{q}}$ is a random matrix and according to complex Gaussian distribution, which indicated the small scale fading channel.and $\mathrm{h}_{\mathrm{sh}, \mathrm{Q}}$ denoted the path loss and large scale fading.Besides, $\mathrm{H}_{\mathrm{w}, \mathrm{q}}$ are independent of $h_{\mathrm{sh}, \mathrm{Q}}$. The large scale fading can be modelled as[6]

$$
h_{\mathrm{sh}, \mathrm{Q}}=\sqrt{\frac{\mathrm{cs}_{\mathrm{q}}}{\mathrm{d}_{q^{\alpha}}}}
$$

where $s_{q}$ is a log-normal shadow fading varible, and $10 \log _{10} s_{q}$ is a zero mean Gaussian random varible with standard deviation $\sigma_{\mathrm{sh}}$. and $d_{q}=1 \mathrm{~km}$ is the distance between the RAU $Q$ and the MS, $\mathrm{c}$ is the constant which is the median of the mean path gain at a reference distance $d_{q}$. where $\alpha$ represent path loss exponent,and it is a chosen parameter in later simulations [6].

In order to analyze and discuss,we suppose that the cell shape is approximated by a circle of radius $K$.so that $\left(K_{\mathrm{Q}}, \theta_{\mathrm{Q}}\right)$ can be expressed the RAUs'polar coordinates relative to the center of the cell and $(\rho, \theta)$ shows the MS's polar coordinate .we assumed the CU/RAU 1 is the center of the cell and the polar coordinates is denoted by $(0,0)$, and the other RAU's polar coordinate are $(3-\sqrt{3}) \mathrm{K} /$

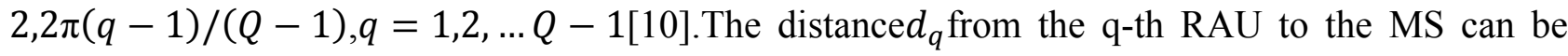
calculated as[6]

$$
d_{q}=\sqrt{\rho^{2}+K_{q}^{2}-2 \rho \cos \left(\theta-\theta_{q}\right)}
$$

And the probability density function $\operatorname{PDF})$ of $(\rho, \theta)$ can be expressed as[6]

$$
\begin{array}{ll}
p(\rho)=\frac{2 \rho}{\mathrm{K}^{2}}, & 0 \leq \rho \leq \mathrm{K}, \\
p(\theta)=\frac{1}{2 \pi}, & 0 \leq \theta \leq 2 \pi .
\end{array}
$$

According to the works[7][13], the power consumption model have been divided the D-MIMO systems into two parts, the idealistic power consumption model and the realistic power consumption model the idealistic power consumption model can be express as

$$
P_{\text {idealistic }}=\frac{P s}{\tau}
$$

where $P_{\mathrm{S}}$ is the transmit power, and the $\tau$ is the radio frequency power amplifier efficiency.

In the pratical application scenarios.we must considering the effects of the other power,so that the realistic power consumption is modeled as [13]

$$
P_{\text {realistic }}=\frac{P_{s}}{\tau}+P_{\text {sta }}+\mathrm{LQ} P_{\text {dyn }}+P_{\mathrm{o}},
$$

where the $P_{\text {sta }}$ is static power, $P_{\text {dyn }}$ is the dynamic power consumption and the $P_{\mathrm{o}}$ is the dissipated power consumption.

The first introduced for EE in [14],Then the research work in [7],so the formula of the EE can be written as 


$$
\eta_{\mathrm{EE}}=\frac{\mathrm{C}}{P_{\text {total }}}
$$

where $C$ denoted the capacity, $P_{\text {total }}$ is the the total power consumption which have two parts and the formula is equal to (7) or (8).

\section{The Capacity and EE of the Distributed MIMO Systems}

In this section,we will first give the expression of the ergodic capacity for the D-MIMO antennas systems, and then shows the expression of outage capacity for the D-MIMO antennas systems.

We consider the reciever have perfect knowledges of channel and unknown at the transmitter.Then,We can get the expression of the ergodic capacity in bits/s/Hz through the mutual information for D-MIMO systems[6]

$$
I=\log _{2} \operatorname{det}\left[\mathrm{I}_{\mathrm{M}}+\frac{P_{S}}{\mathrm{M}} \mathbf{H}^{\mathrm{H}}(\mathbf{d}) \mathbf{H}(\mathbf{d})\right] .
$$

From [6], the mutual information of the outage capacity can be written as

$$
I^{\delta}(\mathbf{d})=\mu_{I}(\mathbf{d})+\sqrt{2 \sigma_{I}^{2}(\mathbf{d})} \operatorname{erfc}^{-1}(2(1-\delta)),
$$

where the $\delta$ is the outage probability, $\mu_{\mathrm{I}}(\mathbf{d})$ and $\sigma_{\mathrm{I}}^{2}$ is the expection and varience of the mutual information,respectively.

In this paper,we consider the outage capacity with $M \leq Q$. For the $(M, N, Q) \mathrm{D}-\mathrm{MIMO}$ systems, the outage capacity can be written as[6]

$$
\mathrm{C}_{\text {out }}=\mu_{I \mathrm{D}-\mathrm{MIMO}}+\sqrt{2 \delta_{\mathrm{ID}-\mathrm{MIMO}}^{2}} \operatorname{erfc}^{-1}(2(1-\delta)) .
$$

From work[6], $\boldsymbol{\mu}_{I}(\mathrm{~d})$ and $\sigma_{\mathrm{I}}(\mathrm{d})$ can be expressed as

$$
\begin{gathered}
\mu_{I \mathrm{D}-\mathrm{MIMO}}(\mathbf{d})=\frac{\mathrm{M}}{\ln 2} \ln \frac{\gamma}{\mathrm{M}}+\frac{\mathrm{M}}{\ln 2}\left(2 \operatorname{lnt} \mathrm{t}_{1}-\frac{1}{2} \ln \mathrm{t}_{2}\right), \\
\delta_{\mathrm{ID}-\mathrm{MIMO}}^{2}(\mathbf{d})=\left(\frac{\mathrm{M}}{\ln 2}\right)^{2}\left(\operatorname{lnt}_{2}-2 \ln \mathrm{t}_{1}\right),
\end{gathered}
$$

where

$$
\gamma=\frac{\mathrm{cPs}}{\mathrm{D}^{\alpha}}
$$

$\gamma$ denoted the noise level of the distributed massive MIMO system refer to distance $K$. where $t_{1}$ and $t_{2}$ can be written as[6]

$$
\begin{gathered}
\mathrm{t}_{1}=\sum_{\mathrm{n}=1}^{\mathrm{N}} \exp \left(\mu_{\mathrm{wn}}+\frac{1}{2} \sigma_{\mathrm{wn}}^{2}\right), \\
\mathrm{t}_{2}=\sum_{\mathrm{n}=1}^{\mathrm{N}} \exp \left(2 \mu_{\mathrm{wn}}+2 \sigma_{\mathrm{wn}}^{2}\right)+2 \sum_{\mathrm{n}=1}^{\mathrm{N}-1} \sum_{\mathrm{m}=\mathrm{n}+1}^{\mathrm{N}} \exp \left(\mu_{\mathrm{wn}}+\mu_{\mathrm{wm}}\right) \\
\times \exp \left(\frac{1}{2} \mu_{\mathrm{wn}}+\frac{1}{2} \sigma_{\mathrm{wm}}^{2}\right) .
\end{gathered}
$$

so,we can get the expression of EE accroding to (8),(9) and (14)

$$
\eta_{\text {D_MIMO_EE_outage }}=\frac{\mu_{I \mathrm{D}-\mathrm{MIMO}^{+}}+\sqrt{2 \delta_{\mathrm{ID}-\mathrm{MIMO}}^{2}} \operatorname{erfc}^{-1}(2(1-\delta))}{P_{\text {total }}} .
$$


The ergodic capacity and outage capacity with $M \geq N L$ case have been discussed and studied in [6],respectively.But the outage capacity with $M \leq L$ without further details.In works[7][11] discussed the EE based on ergodic capacity.Corresponding to the formula(18),we can get some characters for EE which based on outage capacity for the D- MIMO systems.It can be written as in Theorem 1 as following which is demonstrated in Appendix A.

Theorem 1:The EE decreases with the increase of transmit power for the idealistic power consumption model.Nevertheless, the EE of the D-MIMO systems with $M \leq L$ first increases and then decreases under the realistic power consumption model.

Theorem 2: The performance of the EE is increased with the increase of the cell radius, the performance of the EE is decreases with the increase of the outage probability, and the performance of EE increases with the decrease of the path loss exponent.

\section{Numerical Results}

In this section,the numerical results is provided to verify the correctness of the previous expression through Monte Carlo simulation.At first,evaluate the performance of $(1,1,5)$ and $(2,2,7)$ D-MIMO systems under the realistic and idealistic power consumption model,respectively.

Fig.1 As anticipated,the outage capacity is increases with the increase of the transmit power.From the simulation results shown in Fig.2(a), it is seen that the EE decreases with the increase of the idealistic power consumption model.From Fig.2(b),it shows that the EE first increases and then decreases under the realistic power consumption model,This indicates that there is a trade-off between the $\mathrm{EE}$ and the outage capacity or maximum transmit power.

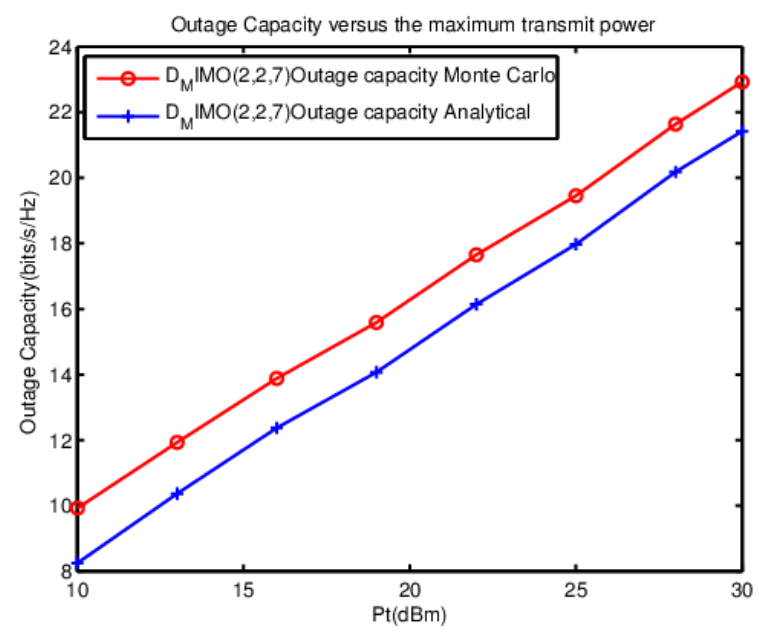

Fig.1.Outage capacity versus the maximum transmit power

From Fig.3,we can see definitely that the EE increases with the increase of the outage probability.Compare with the formula (11) and (12), the outage probability is an important fator to influence the outage capacity, and effect the performance of the energy efficient.Fig. 4 shows that the performance of the EE is decreases with the increase of the cell radius.As in the simulation results, the EE of the cell radius $K=1.5 \mathrm{~km}$ is approximately $30.12 \%$ lower than the EE of the cell radius $K_{2}=1 \mathrm{~km}$. and the EE of the cell radius $K=2 \mathrm{~km}$ is approximately $53.87 \%$ lower than the EE of the cell radius $K_{3}=1 \mathrm{~km}$. From the result shown in Fig. 6 , it is seen that the performance of EE increase with the decrease of the path loss exponent $\alpha$ the EE of the $\alpha=4$ is approximately $8.38 \%$ lower than the $\mathrm{EE}$ of the $\alpha=3$.7. Meanwhile, the EE of the $\alpha=4.3$ is almost $15.139 \%$ lower than the $\mathrm{EE}$ of the $\alpha=3.7$.In conclusion,just agree with the Theorem 2 . 

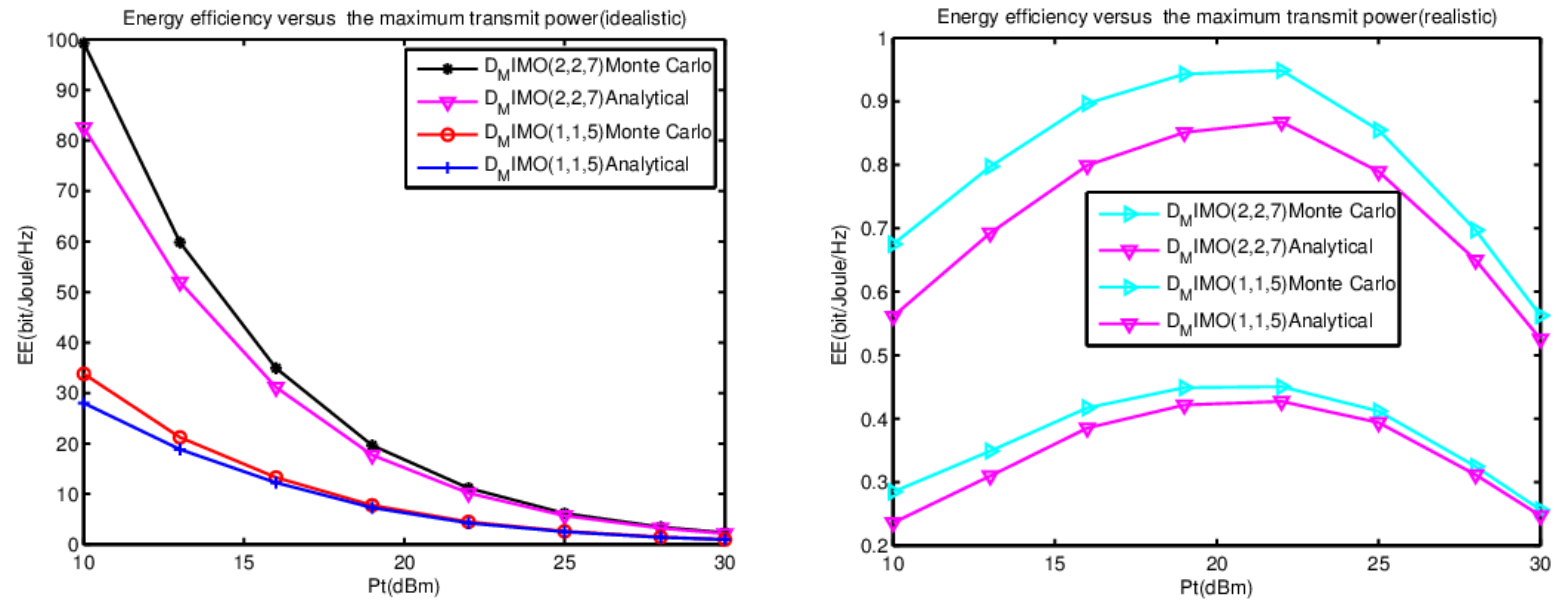

(a)EE under the idealistic power consumption model(b) EE under the realistic power consumption model Fig. 2.EE under the different power consumption model
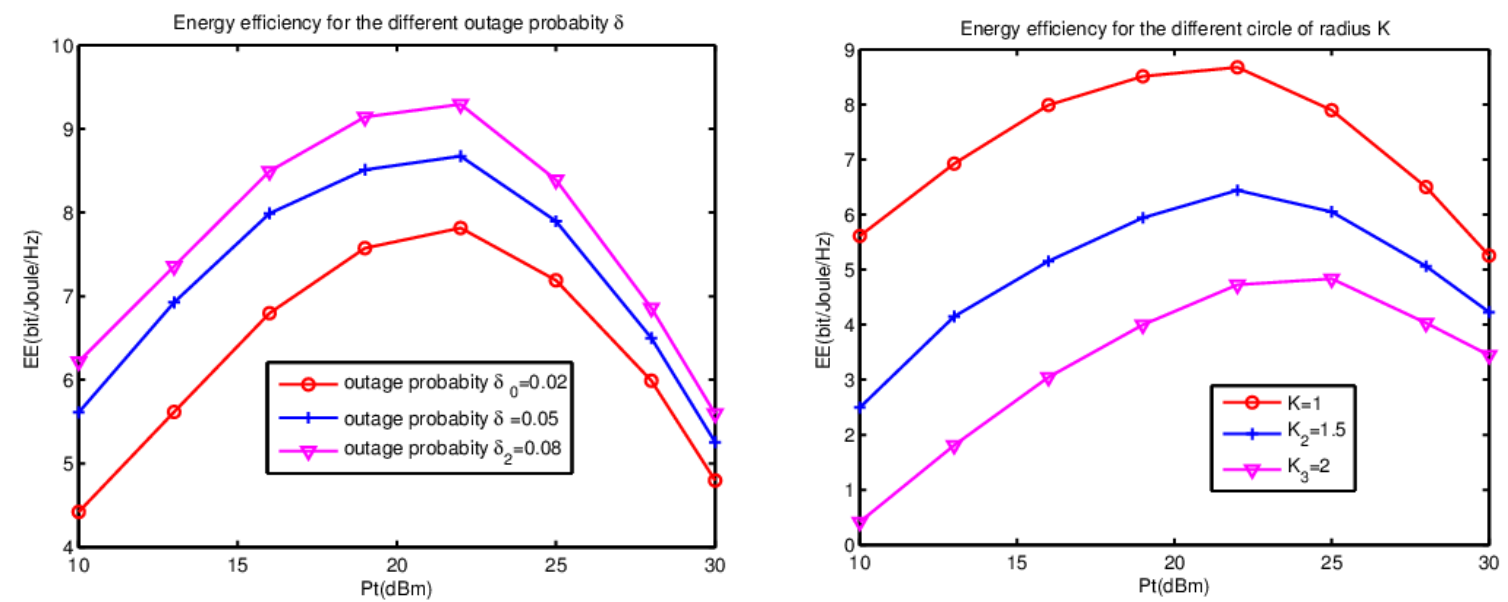

Fig.3. EE versus the maximum transmit power with Fig.4.EE versus the maximumtransmit different outage probability power with different cell radius

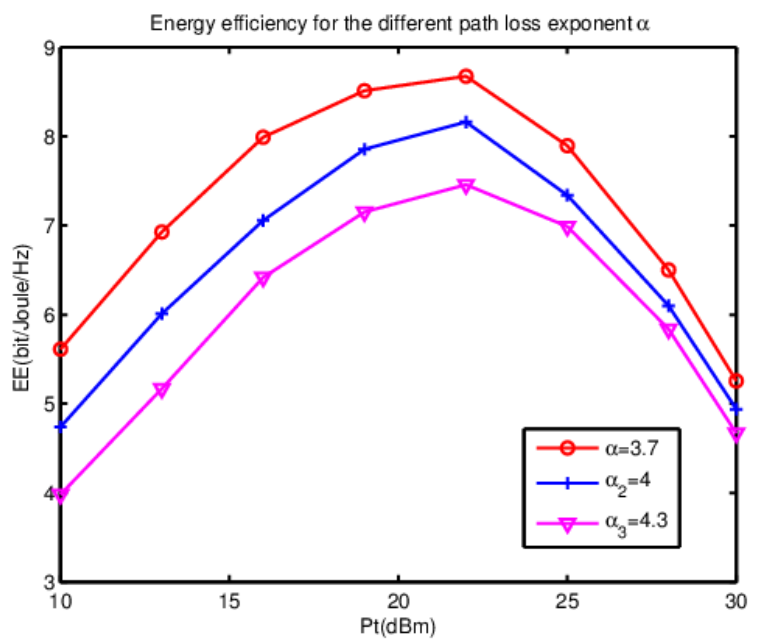

Fig.5.EE versus the maximum transmit power different path loss exponent 


\section{Summary}

In this paper, we investigate the outage capacity and EE in D-MIMO systems. The outage capacity expression and numerical results shown that outage capacity is almost linear with the transmit power,and outage capacity increases with the increase of the transmit power. From the numerical results,we find outage probability,cell radius and path loss exponent will affect the performance of outage capacity.Simulation results have also demonstrated that the EE increases with the increase of the outage probability, the EE increases with the decrease of the cell radius and the EE increases with the decrease of the path loss exponent.

\section{Appendix A: Proof of Theorem 1}

According to (8),(9) and (12),substituting (8) to (12), the EE of the D-MIMO systems can be given as

$$
\eta_{\mathrm{D}_{-} \mathrm{MIMO} \_\mathrm{EE} \_ \text {outage }}=\frac{\mu_{I \mathrm{D}-\mathrm{MIMO}}+\mathrm{V}}{\frac{P_{S}}{\tau}+\mathrm{U}},
$$

where $\mathrm{V}=\sqrt{2 \delta_{\mathrm{ID}-\mathrm{MIMO}}^{2}} \operatorname{erfc}^{-1}(2(1-\delta)), \mathrm{U}=P_{\text {sta }}+\mathrm{LQ} P_{\mathrm{dyn}}+P_{\mathrm{o}}$

After we have to take the derivative of the (19) which based on maximum transmitted power $P_{\mathrm{t}, \text { we get the expression as }}$

$$
\frac{\partial \eta_{\text {D_MIMO_EE_outage }}}{\partial \mathrm{P}_{\mathrm{t}}}=\frac{\frac{\mathrm{M} 1}{\ln 2 \mathrm{Ps}}\left(\frac{\mathrm{Ps}}{\tau}+\mathrm{U}\right)-\left(\frac{\mathrm{M}}{\ln 2} \ln \frac{\mathrm{cPs}}{\mathrm{D}^{\alpha}}+\mathrm{V}\right)\left(\frac{1}{\tau}\right)}{\left(\frac{\mathrm{Ps}}{\tau}+\mathrm{U}\right)^{2}} .
$$

Obviously,the denominator of the formula (20) is a positive quality.So we focus on the molecular researching,and then we denoted molecular be a new function

$$
\mathrm{g}(P \mathrm{~s})=\frac{\mathrm{M}}{\ln 2} \frac{1}{\mathrm{Ps}}\left(\frac{P s}{\tau}+\mathrm{U}\right)-\left(\frac{\mathrm{M}}{\ln 2} \ln \frac{\mathrm{cPs}}{\mathrm{D}^{\alpha}}+\mathrm{V}\right)\left(\frac{1}{\tau}\right) .
$$

We have to take the first derivative of (21) based on $P_{S}$, and get the equation as

$$
\frac{\partial \mathrm{g}(P s)}{\partial P S}=-\frac{\mathrm{M}}{\ln 2}\left(\frac{\mathrm{U}}{P s^{2}}+\frac{1}{\tau P S}\right)<0 .
$$

It is shows that the function (22) decreases with the increase of transmit power $P_{S}$,so we simply drawed $\mathrm{g}(\infty) \leq \mathrm{g}\left(P_{S}\right) \leq \mathrm{g}(0)$. so we can get the conclusion from the (22) which $\lim _{\mathrm{Pt} \rightarrow 0} \mathrm{~g}\left(P_{S}\right)>0$ and $\lim _{\mathrm{Pt} \rightarrow \infty} \mathrm{g}\left(P_{S}\right)>0$ is that EE of D-MIMO systems based on outage capacity first increases and then decreases with the increase of power $P_{S}$. Theorem 1 is proved.

\section{Acknowledgement}

This work is partially supported by the Natural Science Foundation of China under grants 61601300, the Science and Technology In-novation Comm-ission of Shenzhen under Grants JCYJ20150324140036835, the OpenResearch Fund through the National Mobi-le Communications Research Lab-oratory, Southeast University, Nanjing, China, under Grant 2017D10, Shenzhen University start-up funding No. 2016053.

\section{References}


[1]D. Gesbert, M. Shafi, D. Shiu, P. J. Smith, and A.Naguib,"From theory to practice: An overview of MIMO spacetimen coded wireless systems," IEEE J. Select. Areas Commun.,vo 1. 21, no. 3, pp. 281-302, Apr. 2003.

[2]A. Goldsmith, S. A. Jafar, N. Jindal, and S. Vishwanath, “Capacity Limits of MIMO Channels”, IEEE J. Select.Areas Commun., vol. 21, no. 5, pp. 684-702, June 2003.

[3]H. Yin, D. Gesbert, M. Filippou, and Y. Liu, "A coordinated approach to channel estimation in large-scale multiple-antenna systems," IEEE J. Sel.Areas Commun., vol. 31, no. 2, pp. 264-273, Feb. 2013.

[4]H. Q. Ngo and E. G. Larsson, "EVD-based channel estimations for multicell multiuser MIMO with very large antenna arrays," in Proc. IEEE ICASSP, Mar. 2012.

[5]W. Roh and A. Paulraj, "MIMO channel capacity for the distributed antenna systems," in IEEE Veh. Technology Conf. (VTC’02), vol. 3, Sept.2002, pp. 1520-1524.

[6]D.-M. Wang ,X.-H.Y,J.-Z.W,Y.W, and X.-Y.Hou, "Spectral efficiency of distributed MIMO systems, " IEEE J. Sel. Areas Commun., vol. 31, pp. 2112-2127, Oct. 2013.

[7]C.-L.He,G.-Y.Li,F.-C.Zheng, and X-H.You,"Energy efficiency of distributed MIMO systems."in proc.IEEE S.I.P,Sept. 2014:218-222.

[8]Z.-D.Wang, Giannakis, and G.B. "Outage mutual information of space-time MIMO channels." IEEE Trans. Inf. Theory ,50.4(2004):657-662.

[9]Zappone, Alessio, E. Jorswieck, and A. Leshem. "Distributed Resource Allocation for Energy Efficiency in MIMO OFDMA Wireless Networks." IEEE J.Select.Areas Commun., pp.99(2016):1-1.

[10]C.-L.He,J.-J.Yin,Y.-J.He,M.Huang, and B.Zhao,"Energy efficiency of distributed massive MIMO systems." J. Commun. Net., 18.4 (2016): 649-657.

[11]Y. Tokgoz, and B.D. Rao. "Outage probability of multi-cellular MIMO systems in Rayleigh fading." IEEE Int.Con.Acou, S.S.P, 2004. Proceedings IEEE, 2004:ii-5-8 vol.2.

[12]Z. Wang and G. B. Giannakis, "Outage mutual information of spacetime MIMO channels, " IEEE Trans. Inf. Theory, vol. 50, no. 4, pp.657-662, Apr. 2004.

[13]C.-L. He, G. Y. Li, F.-C. Zheng, and X.-H. You, "Energy-efficient resource allocation in OFDM systems with distributed antennas," IEEE Trans. Veh.Technol., vol. 63, pp. 1223-1231, Mar. 2013.

[14]Kwon, Hyuck, and T. G. Birdsall. "Channel capacity in bits per joule." IEEE J.Oce.Eng., 11.1(1986):97-99. 